\title{
Short-term reduction in intrinsic heart rate during biventricular pacing after cardiac surgery: A substudy of a randomized clinical trial
}

\author{
Jiajie G. Lu, BA, ${ }^{\mathrm{a}}$ Anthony Pensiero, ${ }^{\mathrm{a}}$ Linda Aponte-Patel, MD, ${ }^{\mathrm{b}}$ Bryan Velez de Villa, BS, ${ }^{\mathrm{a}}$ \\ Alexander Rusanov, MD, ${ }^{\mathrm{c}}$ Bin Cheng, PhD, ${ }^{\mathrm{d}}$ Santos E. Cabreriza, MBA, ${ }^{\mathrm{a}}$ and Henry M. Spotnitz, MD ${ }^{\mathrm{a}}$
}

\begin{abstract}
Background: The Biventricular Pacing After Cardiac Surgery trial investigates hemodynamics of temporary pacing in selected patients at risk of left ventricular dysfunction. This trial demonstrates improved hemodynamics during optimized biventricular pacing compared with atrial pacing at the same heart rate 1 and 2 hours after bypass and reduced vasoactive-inotropic score over the first 4 hours after bypass. However, this advantage of biventricular versus atrial pacing disappears 12 to 24 hours later. We hypothesized that changes in intrinsic heart rate can explain variable effects of atrial pacing in this setting.
\end{abstract}

\begin{abstract}
Methods: Heart rate, mean arterial pressure, cardiac output, and medications depressing heart rate were analyzed in patients randomized to continuous biventricular pacing $(n=16)$ or standard of care $(n=18)$.

Results: During 30-second testing periods without pacing, intrinsic heart rate was lower in the paced group 12 to 24 hours after bypass $(76.5 \pm 17.5$ vs $91.7 \pm 13.0$ beats per minute; $P=.040)$ but not 1 or 2 hours after bypass. Cardiac output ( $4.4 \pm 1.2$ vs $3.6 \pm 1.9 \mathrm{~L} / \mathrm{min} ; P=.054)$ and stroke volume $(53 \pm 2$ vs $42 \pm 2 \mathrm{~mL} ; P=.051)$ increased overnight in the paced group. Vasoactive medication doses were not different between groups, whereas dexmedetomidine administration was prolonged over postoperative hours 12 to 24 in the paced group $(793 \pm 528$ vs $478 \pm 295$ minutes; $P=.013)$.
\end{abstract}

Conclusions: These observations suggest that hemodynamic benefits of biventricular pacing 12 to 24 hours after cardiopulmonary bypass lead to withdrawal of sympathetic drive and decreased intrinsic heart rate. Depression of intrinsic rate increases the apparent benefit of atrial pacing in the chronically paced group but not in the control group. Additional study is needed to define clinical benefits of these effects. (J Thorac Cardiovasc Surg 2013;146:1494-500)

Biventricular pacing (BiVP) is beneficial for patients with heart failure characterized by ventricular dyssynchrony. ${ }^{1}$ BiVP can induce resynchronization, with function optimized by adjusting the length of both atrioventricular delay (AVD) and interventricular delay (VVD). BiVP reduces morbidity and mortality and improves quality of life and walking ability for patients with mild to severe heart failure who exhibit a prolonged QRS duration (QRSd) of

From the Departments of Surgery, ${ }^{\mathrm{a}}$ Pediatrics, ${ }^{\mathrm{b}}$ and Anesthesiology, ${ }^{\mathrm{c}}$ Columbia University, New York, NY; and the Department of Biostatistics, ${ }^{\mathrm{d}}$ Mailman School of Public Health, New York, NY.

Supported by National Institutes of Health T35 Training Grant (J.G.L.) and grant RO1 HL080152 (H.M.S.)

Disclosures: Dr Spotnitz is the founder of and a consultant (volunteer) for Strategic Pacing Systems LLC, and has equity interest in and is on the Scientific Advisory Board of Biophan Technologies. Mr Cabreriza is an unpaid consultant for Strategic Pacing Systems LLC. All other authors have nothing to disclose with regard to commercial support.

Dr Spotnitz is the George H. Humphreys, II, Professor of Surgery.

Received for publication Oct 5, 2012; revisions received March 29, 2013; accepted for publication June 27, 2013; available ahead of print Sept 30, 2013.

Address for reprints: Henry M. Spotnitz, MD, Department of Surgery, Columbia University Medical Center, 622 W 168th St, 10th Floor, Vanderbilt Clinic, Room 1010, New York, NY 10032 (E-mail: hms2@ columbia.edu).

$0022-5223 / \$ 36.00$

Copyright $(9) 2013$ by The American Association for Thoracic Surgery

http://dx.doi.org/10.1016/j.jtcvs.2013.06.056 greater than 120 milliseconds and a left ventricular ejection fraction (LVEF) of $35 \%$ or less. ${ }^{2-5}$ In addition, BiVP, with or without an intracardiac defibrillator, can reduce mortality and hospitalizations after implantation. ${ }^{3,6}$ Mechanistically, BiVP has improved LVEF and reversed left ventricular (LV) remodeling characteristic of advanced heart failure. ${ }^{5,7}$ More important, BiVP can increase contractility without increasing myocardial oxygen demand. ${ }^{8}$

BiVP may be beneficial for patients after open heart surgery (OHS). Previous studies that investigated temporary BiVP after OHS have used a primary eligibility criterion of low preoperative LVEF, which can independently predict risk of acute heart failure after OHS. ${ }^{9}$ These studies have shown mixed results but suggest that BiVP is most effective immediately after cardiopulmonary bypass (CPB). ${ }^{10-13}$ Complicating these results is that up to $30 \%$ of patients do not respond to permanent BiVP. ${ }^{2,14}$ Optimization of permanent BiVP pacing settings can decrease this nonresponse rate, ${ }^{15,16}$ but the optimal settings for temporary BiVP after OHS are unknown.

The Biventricular Pacing after Cardiac Surgery (BiPACS) trial is a randomized, controlled study of temporary, optimized BiVP for patients undergoing OHS. 


$$
\begin{aligned}
& \text { Abbreviations and Acronyms } \\
& \begin{aligned}
\text { AAI } & =\text { atrial pacing } \\
\text { AVD } & =\text { atrioventricular delay } \\
\text { BiPACS } & \text { Biventricular Pacing after Cardiac } \\
& \text { Surgery } \\
\text { BiVP } & =\text { biventricular pacing } \\
\text { bpm } & =\text { beats per minute } \\
\mathrm{CO} & =\text { cardiac output } \\
\mathrm{CPB} & =\text { cardiopulmonary bypass } \\
\mathrm{ICU} & =\text { intensive care unit } \\
\mathrm{LVEF} & =\text { left ventricular ejection fraction } \\
\mathrm{MAP} & =\text { mean arterial pressure } \\
\mathrm{OHS} & =\text { open heart surgery } \\
\text { QRSd } & =\text { QRS duration } \\
\text { SOC } & =\text { standard of care } \\
\text { VIS } & =\text { vasoactive-inotrope score } \\
\text { VVD } & =\text { interventricular delay }
\end{aligned}
\end{aligned}
$$

Enrollemnt criteria are described in Methods. The primary end point for this study is cardiac index, measured by thermodilution in the intensive care unit (ICU). In addition, the BiPACS trial tests optimization of BiVP for all enrolled patients at 3 time points: immediately after CPB (phase I), after chest closure (phase II), and 12 to 24 hours postoperatively (phase III). At phase I in the BiPACS trial, optimized BiVP increased cardiac output (CO) by $13 \%$ compared with no pacing, whereas atrial pacing (AAI) at the same heart rate provided no benefit. ${ }^{17}$ Interestingly, in phase II, the effect of AAI was intermediate between no pacing and optimized $\mathrm{BiVP}^{18}$; and in phase III, the benefit of AAI was indistinguishable from BiVP. ${ }^{18}$ These data indicate that BiVP increases stroke volume immediately after CPB (phase I), whereas the benefit of BiVP and AAI in the ICU (phase III) is primarily due to an increase in heart rate.

Atrial pacing is, thus, increasingly effective from phase I to phase III, but the mechanism for this trend is undefined. We hypothesized that this increasing AAI efficacy might be related to changes in intrinsic heart rate over time after $\mathrm{CPB}$. The pacing rate in $\mathrm{AAI}$ and BiVP modes was 90 beats per minute (bpm) or $10 \mathrm{bpm}$ higher than the patient's intrinsic heart rate if the intrinsic rate exceeded $90 \mathrm{bpm}$. Thus, a decrease in intrinsic heart rate could augment the percentage increase in heart rate during AAI pacing, in turn increasing the fractional change in cardiac output.

This substudy analyzes changes in intrinsic heart rate and related variables in phases I, II, and III of the BiPACS trial. Because enrollment in the trial is complete, we are also able to compare these variables across randomization groups.

\section{METHODS \\ BiPACS Study Population}

The BiPACS protocol is approved by the Columbia University Medical Center Institutional Review Board and supported by the National Institutes of Health under an investigational device exception from the Food and Drug Administration (No. G050189). The protocol has been described in detail previously. ${ }^{17}$ Adult patients undergoing elective surgery on CPB are screened for inclusion in the BiPACS trial by trained study coordinators and investigators, with permission from the attending surgeon. All patients in the study give written, informed consent. Eligibility criteria include preoperative congestive heart failure, an LVEF of $40 \%$ or lower and a QRSd of 100 milliseconds or greater, or combined mitral and aortic valve replacement. Patients are excluded for atrial fibrillation, second- or third-degree AV block, congenital heart disease, intracardiac shunts, or heart rate greater than $120 \mathrm{bpm}$ after CPB.

\section{Study Design and Protocol}

All BiPACS patients undergo BiVP optimization during phases I, II, and III, defined above. Randomization is done after phase I. In addition, patients in the BiVP group are paced continuously between phases I and III. The primary end point is cardiac index measured by thermodilution in the ICU. The present study does not examine primary end point data.

In phase I, 38 settings of BiVP with varying AVD, VVD, and ventricular placing sites are tested in randomized order to determine an optimal BiVP protocol designated $\mathrm{P} 1$, optimized with an aortic flow probe. P1 is tested against AAI at the same heart rate and against the patient's intrinsic sinus rhythm at the end of phase I.

In phase II, BiVP settings are again tested in a different randomized order to determine a second optimal BiVP protocol, P2. P1 and P2 are then compared against each other and against AAI and no pacing, at the end of phase II. Mean arterial pressure (MAP) is a surrogate marker for $\mathrm{CO}$ in phase II, because chest closure precludes use of flow probes and time constraints obviate use of thermodilution.

Patients in the BIVP group are paced from the end of phase I to the start of phase II under protocol P1. The BIVP group is then paced from phase II to phase III with the optimum phase II protocol (either $\mathrm{P} 1$ or P2), as determined using MAP. Patients in the standard-of-care (SOC) group are not paced between phases. The primary end point at the start of phase III is CO by thermodilution, using a Swan-Ganz catheter.

At the start of phase III, the active pacing protocol is again compared with AAI and no pacing using thermodilution CO; 212 settings of AVD and VVD are then tested in random order. The 10 settings yielding the highest MAP are retested to determine an optimal phase III setting, P3. P3 is finally compared with AAI and no pacing by thermodilution $\mathrm{CO}$.

\section{Data Analysis}

At all phases, electrocardiographic and arterial pressure tracings are recorded. In phase I, flow velocity is recorded using an aortic flow probe. Data are converted to digital form with a PowerLab AD system (ADInstruments, Inc, Colorado Springs, Colo) and stored on a personal computer (Apple Computer, Inc, Cupertino, Calif) with MacLab software (ADInstruments, Inc). Data are then loaded into Matlab (The MathWorks, Inc, Natick, Mass), where heart rate, CO, and MAP are averaged and recorded over one respiratory cycle toward the end of no pacing, AAI, and optimized BiVP segments. ${ }^{17,18}$ Doses of vasoactive medications and duration of sedative infusions are obtained from the Eclipsys patient record system (Allscripts Healthcare Solutions, Inc, Chicago, Ill) at New YorkPresbyterian Hospital. Vasoactive-inotrope scores were calculated as described in a prior substudy ${ }^{22}$ : 
VIS $=$ Dopamine Dose $(\mu \mathrm{g} / \mathrm{kg}$ per minute $)$

+ Dobutamine Dose $(\mu \mathrm{g} / \mathrm{kg}$ per minute $)$

$+100 \times$ Epinephrine Dose $(\mu \mathrm{g} / \mathrm{kg}$ per minute $)$

$+10 \times$ Milrinone Dose $(\mu \mathrm{g} / \mathrm{kg}$ per minute $)$

$+10,000 \times$ Vasopressin Dose $(\mathrm{U} / \mathrm{kg}$ per minute $)$

$+100 \times$ Norepinephrine Dose $(\mu \mathrm{g} / \mathrm{kg}$ per minute $)$

$+10 \times$ Phenylephrine Dose $(\mu \mathrm{g} / \mathrm{kg}$ per minute $)$

These data were then evaluated for changes and differences in intrinsic heart rate, $\mathrm{CO}, \mathrm{MAP}$, and drug administration times. To determine whether there was an increasing or decreasing trend in heart rate between phases, paired $t$-tests were conducted, with $P<.05$ considered significant. To determine differences between BiVP and SOC groups, 2-sample $t$-tests were conducted, with $P<.05$ again considered significant. Statistical analysis was performed using SAS 9.1 (SAS Institute, Inc, Cary, NC).

\section{RESULTS}

\section{Study Population}

Table 1 displays the average baseline characteristics (mean $\pm \mathrm{SD}$ ) of 34 patients examined in this substudy, 16 in the BiVP group and 18 in the SOC group. There were no significant differences between the BiVP and SOC groups in age $(71 \pm 8$ vs $68 \pm 11$ years; $P=.35$ ), sex (males, $81 \%$ vs $78 \% ; P=.80$ ), weight $(70 \pm 14$ vs $78 \pm 15 \mathrm{~kg} ; P=.14)$, preoperative LVEF $(35.6 \% \pm 12.8 \%$ vs $30.7 \% \pm 16.4 \% ; P=.34)$. There was also no significant difference between the groups in the type of surgery based on the number of coronary artery bypass grafts $\left(\chi^{2}=6.39, P=.17\right)$, or the number of aortic valve replacements or repairs $\left(\chi^{2}=2.24, P=.13\right)$. The BiVP group had more mitral valve replacements or repairs compared with SOC patients (13/16 vs 7/18

TABLE 1. Patient baseline characteristics

\begin{tabular}{lccc}
\hline \multicolumn{1}{c}{ Characteristic } & $\begin{array}{c}\text { Randomized to } \\
\text { BiVP }(\mathbf{n}=\mathbf{1 6})\end{array}$ & $\begin{array}{c}\text { Randomized to } \\
\text { SOC }(\mathbf{n}=\mathbf{1 8})\end{array}$ & $\begin{array}{c}\boldsymbol{P} \\
\text { value }\end{array}$ \\
\hline Age, y & $70.8 \pm 7.5$ & $67.6 \pm 11.4$ & .35 \\
Male sex, \%* & 81 & 78 & .80 \\
Weight, kg & $70.1 \pm 14.4$ & $77.7 \pm 14.9$ & .14 \\
Preoperative LVEF, \% & $35.6 \pm 12.8$ & $30.7 \pm 16.4$ & .34 \\
Preoperative QRS, ms & $116.5 \pm 21.2$ & $120.1 \pm 23.1$ & .64 \\
Bypass time, min & $163.6 \pm 73.3$ & $128.5 \pm 35.6$ & .08 \\
Crossclamp time, min & $102.8 \pm 53.1$ & $81.6 \pm 39.5$ & .21 \\
Type of surgery & & & \\
CABG $(0,1,2,3,4)^{*}, \dagger$ & $7,2,3,0,3$ & $6,1,2,6,3$ & .17 \\
AVR/r $(0, \mathrm{R}, \mathrm{r})^{*}, \ddagger$ & $4,12,0$ & $9,9,0$ & .13 \\
MVR/r $(0, \mathrm{R}, \mathrm{r}) *, \S$ & $3,7,6$ & $11,3,4$ & .04 \\
\hline
\end{tabular}

Data are given as mean \pm SD unless otherwise indicated. BiVP, Biventricular pacing; $S O C$, standard of care; $L V E F$, left ventricular ejection fraction; $C A B G$, coronary artery bypass grafting; $A V R / r$, aortic valve replacement/repair; $M V R / r$, mitral valve replacement/repair; $Q R S$, duration of QRS complex on electrocardiogram, in milliseconds. *Statistical significance calculated through $\chi^{2}$ test. $\dagger$ Shown as number of patients with $0,1,2,3,4$ bypasses. ‡Shown by number of patients with no repair, replacement, or repair to the aortic valve. §Shown by number of patients with no repair, replacement, or repair to the mitral valve. patients; $\left.\chi^{2}=6.48, P=.04\right)$. There was a trend toward increased duration of CPB in the BiVP group (164 \pm 73 vs $128 \pm 36$ minutes; $P=.08$ ), but no significant differences in crossclamp time (103 \pm 53 vs $82 \pm 40$ minutes; $P=.21)$.

\section{Clinical Results}

Figure 1 presents average intrinsic heart rates at the end of phases I and II and the beginning of phase III. These data were obtained during comparisons between BiVP, AAI, and no pacing. Compared with SOC patients, heart rate was significantly lower in BiVP patients in phase III (76.5 \pm 17.5 vs $91.7 \pm 13.0 \mathrm{bpm} ; P=.040)$, but not significantly different in phase I ( $77.2 \pm 19.7$ vs $78.3 \pm 14.7 \mathrm{bpm}$; $P=.87)$ and phase II $(83.1 \pm 14.1$ vs $86.0 \pm 18.7 \mathrm{bpm}$; $P=.67)$. There was a trend toward decreasing intrinsic heart rate from phase II to phase III in the BiVP group (76.5 \pm 17.5 vs $86.0 \pm 18.7 \mathrm{bpm} ; P=.063)$, but not in SOC patients $(83.1 \pm 14.1$ vs $91.7 \pm 13.0 \mathrm{bpm} ; P=.45)$.

Table 2 presents average hemodynamics for all patients studied. From phase I to phase III, there was an upward trend in BiVP patients of $\mathrm{CO}(4.4 \pm 1.2 \mathrm{vs} 3.6 \pm 1.9 \mathrm{~L} /$ $\min ; P=.054)$ and stroke volume $(53.4 \pm 17.3$ vs $42.5 \pm$ $21.4 \mathrm{~mL} ; P=.051$ ) (Figure 2). There was no significant change in MAP in the BiVP group from phase I to phase II (76.4 \pm 9.5 vs $74.6 \pm 12.2 \mathrm{~mm} \mathrm{Hg} ; P=.76)$, or phase II to phase III $(74.6 \pm 12.2$ vs $79.9 \pm 12.8 \mathrm{~mm} \mathrm{Hg}$; $P=.15)$. In the SOC group, there was no significant increase in $\mathrm{CO}(5.1 \pm 1.1$ vs $4.8 \pm 1.3 \mathrm{~L} / \mathrm{min} ; P=.14)$ or stroke volume $(55.9 \pm 14.1$ vs $55.5 \pm 14.7 \mathrm{~mL}$; $P=.95$ ) between phases I and III. There were no significant changes in MAP for SOC patients from phase I to phase II $(69.9 \pm 8.7$ vs $74.5 \pm 11.5 \mathrm{~mm} \mathrm{Hg} ; P=.16)$ and

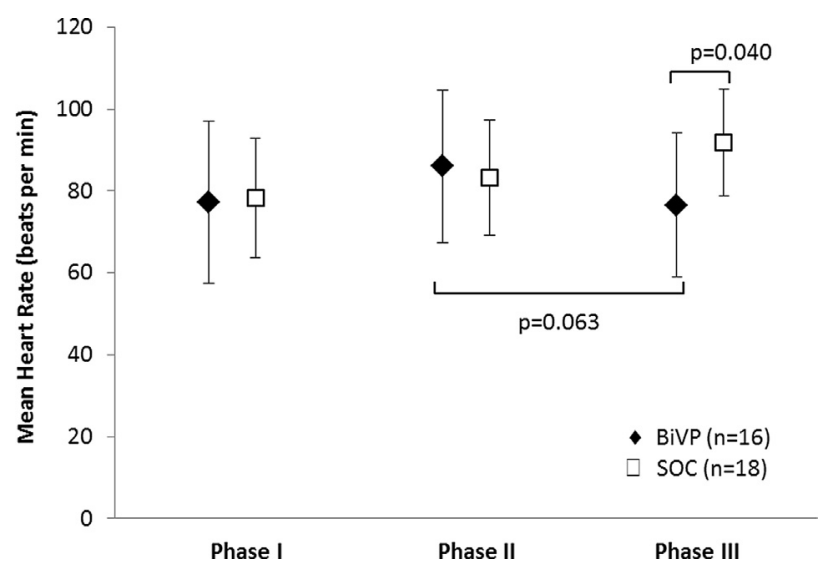

FIGURE 1. Mean intrinsic heart rates for biventricular pacing (BiVP) $(\mathrm{n}=16)$ and standard-of-care $(S O C)(\mathrm{n}=18)$ groups from phase I to phase III. Brackets indicate $P$ values. Intrinsic heart rate in the BiVP group was significantly lower than in the SOC group in phase III. A trend of decreasing heart rates in BiVP patients from phase II to phase III is not statistically significant $(P=.0631)$. Data from Table 2 . 
TABLE 2. Hemodynamics of BiPACS patients in phases I to III

\begin{tabular}{|c|c|c|c|c|c|c|c|c|c|c|c|}
\hline & & & Pha & ase I & & Pha & ase II & & Phas & se III & \\
\hline Setting & & HR, bpm & $\begin{array}{c}\text { MAP, } \\
\text { mm Hg }\end{array}$ & $\begin{array}{c}\text { CO, } \\
\text { L/min }\end{array}$ & $\mathrm{SV}, \mathrm{mL}$ & HR & MAP & HR & MAP & $\mathrm{CO}$ & SV \\
\hline $\begin{array}{c}\text { No. of } \\
\text { patients } \\
\end{array}$ & 16 & 12 & 12 & 12 & 12 & 10 & 10 & 13 & 13 & 13 & 13 \\
\hline $\begin{array}{l}\text { BiVP } \\
\quad(\mathrm{n}=16)\end{array}$ & $\begin{array}{l}\text { NoP } \\
\text { AAI } \\
\text { BiVP }\end{array}$ & $\begin{array}{l}77.2 \pm 19.7 \\
96.5 \pm 11.0 \\
96.3 \pm 10.6 \\
\end{array}$ & $\begin{aligned} & 76.4 \pm 9.5 \\
& 79.3 \pm 9.4 \\
& 79.9 \pm 12.0 \\
&\end{aligned}$ & $\begin{array}{l}3.56 \pm 1.85 \\
3.65 \pm 1.82 \\
4.08 \pm 2.19 \\
\end{array}$ & $\begin{array}{l}42.5 \pm 21.4 \\
37.4 \pm 18.1 \\
42.7 \pm 22.9 \\
\end{array}$ & $\begin{array}{l}86.0 \pm 18.7 \\
98.0 \pm 12.0 \\
95.8 \pm 11.0 \\
\end{array}$ & $\begin{array}{l}74.6 \pm 12.2 \\
75.7 \pm 11.9 \\
78.8 \pm 13.4 \\
\end{array}$ & $\begin{array}{l}76.5 \pm 17.5 \\
94.8 \pm 13.0 \\
94.8 \pm 13.0\end{array}$ & $\begin{array}{l}79.9 \pm 12.8 \\
88.3 \pm 12.6 \\
85.6 \pm 12.5 \\
\end{array}$ & $\begin{array}{l}4.40 \pm 1.17 \\
4.89 \pm 1.19 \\
4.86 \pm 1.54 \\
\end{array}$ & $\begin{array}{l}53.4 \pm 17.3 \\
50.6 \pm 14.4 \\
49.1 \pm 14.5 \\
\end{array}$ \\
\hline $\begin{array}{c}\text { No. of } \\
\text { patients }\end{array}$ & 18 & 18 & 18 & 18 & 18 & 14 & 14 & 9 & 9 & 9 & 9 \\
\hline $\begin{array}{l}\text { SOC } \\
\qquad(\mathrm{n}=18)\end{array}$ & $\begin{array}{l}\text { NoP } \\
\text { AAI } \\
\text { BiVP }\end{array}$ & $\begin{array}{l}78.3 \pm 14.7 \\
92.3 \pm 10.1 \\
95.8 \pm 7.4\end{array}$ & $\begin{array}{l}69.9 \pm 8.7 \\
71.8 \pm 11.0 \\
76.4 \pm 14.1\end{array}$ & $\begin{array}{l}4.79 \pm 1.33 \\
4.54 \pm 1.56 \\
5.17 \pm 1.60\end{array}$ & $\begin{array}{l}55.9 \pm 14.1 \\
49.2 \pm 17.2 \\
54.8 \pm 18.7\end{array}$ & $\begin{array}{l}83.1 \pm 19.1 \\
96.1 \pm 11.0 \\
97.0 \pm 9.2\end{array}$ & $\begin{array}{l}74.5 \pm 11.5 \\
75.9 \pm 13.1 \\
77.4 \pm 10.1\end{array}$ & $\begin{array}{r}91.7 \pm 13.0 \\
98.5 \pm 8.4 \\
100.2 \pm 9.5\end{array}$ & $\begin{array}{l}74.5 \pm 11.0 \\
81.4 \pm 11.2 \\
82.2 \pm 10.9\end{array}$ & $\begin{array}{l}5.05 \pm 1.11 \\
5.45 \pm 1.17 \\
5.14 \pm 1.12\end{array}$ & $\begin{array}{l}55.5 \pm 14.7 \\
56.2 \pm 13.4 \\
51.4 \pm 13.1\end{array}$ \\
\hline $\begin{array}{l}\text { All } \\
\qquad(\mathrm{N}=34)\end{array}$ & $\begin{array}{l}\text { NoP } \\
\text { AAI } \\
\text { BiVP }\end{array}$ & $\begin{array}{l}77.9 \pm 16.6 \\
94.2 \pm 10.5 \\
96.0 \pm 8.8\end{array}$ & $\begin{array}{l}72.5 \pm 9.4 \\
75.1 \pm 10.8 \\
78.0 \pm 13.2\end{array}$ & $\begin{array}{l}4.31 \pm 1.64 \\
4.15 \pm 1.70 \\
4.68 \pm 1.94\end{array}$ & $\begin{array}{l}48.8 \pm 19.1 \\
44.0 \pm 18.2 \\
49.4 \pm 21.2\end{array}$ & $\begin{array}{l}84.3 \pm 15.9 \\
96.9 \pm 11.2 \\
96.5 \pm 9.8\end{array}$ & $\begin{array}{l}74.6 \pm 11.5 \\
75.1 \pm 12.3 \\
77.8 \pm 11.5\end{array}$ & $\begin{array}{l}82.7 \pm 17.3 \\
96.3 \pm 11.3 \\
97.2 \pm 11.7\end{array}$ & $\begin{array}{l}77.9 \pm 12.1 \\
85.5 \pm 12.3 \\
84.1 \pm 11.7\end{array}$ & $\begin{array}{l}4.69 \pm 1.17 \\
5.13 \pm 1.19 \\
4.98 \pm 1.35\end{array}$ & $\begin{array}{l}54.4 \pm 15.6 \\
52.9 \pm 14.0 \\
50.1 \pm 13.7\end{array}$ \\
\hline
\end{tabular}

Data are given as mean \pm SD. BiPACS, Biventricular Pacing after Cardiac Surgery; $H R$, heart rate; $b p m$, beats per minute; $M A P$, mean arterial pressure; $C O$, cardiac output; $S V$, stroke volume; $B i V P$, biventricular pacing; $N o P$, no pacing; $A A I$, atrial pacing; $S O C$, standard of care.

phase II to phase III $(74.5 \pm 11.5$ vs $75.0 \pm 11.0 \mathrm{~mm} \mathrm{Hg}$; $P=.93)$.

Figure 3 presents the relation of percentage changes in heart rate and $\mathrm{CO}$ during AAI pacing in phase III. There is a significant relationship between these variables for both the BiVP patients $(P=.002)$ and all patients $(P=.005)$, in which percentage change in heart rate is roughly twice the percentage change in $\mathrm{CO}$.

Figure 4 and Table 3 present the average vasoactiveinotrope score (VIS) of both BiVP and SOC patients at all 3 phases. VIS trended lower in BiVP than SOC patients in phase $\mathrm{I}(10.9 \pm 8.0$ vs $13.3 \pm 10.9 ; P=.502)$,

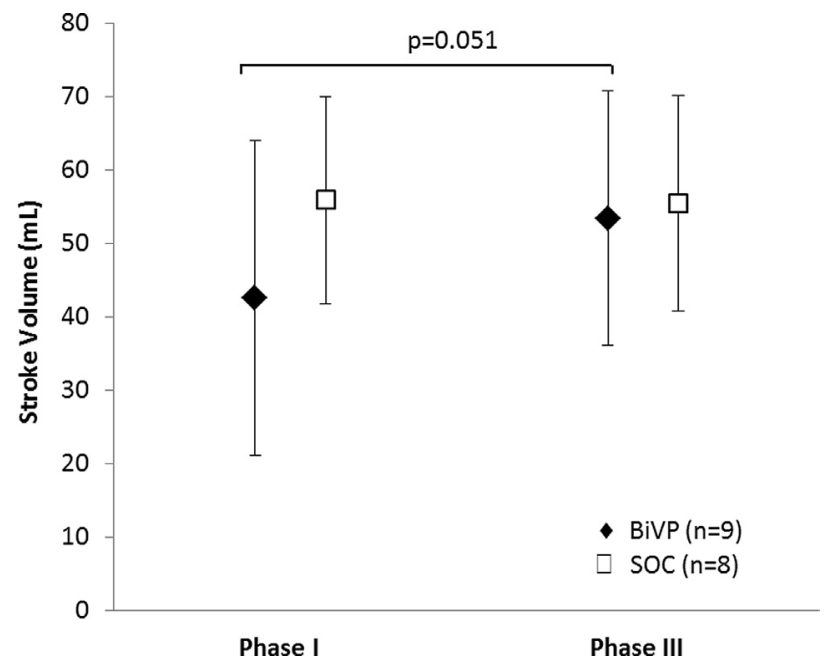

FIGURE 2. Mean stroke volume $(S V)$ without pacing for biventricular pacing $(B i V P)$ and standard-of-care (SOC) groups at phase I and phase III. Trend toward increasing SV in the BiVP group $(P=.051)$. Data from Table 2. phase II $(9.5 \pm 6.7$ vs $13.2 \pm 10.8 ; P=.277)$, and phase III $(7.5 \pm 7.4$ vs $8.4 \pm 9.2 ; P=.776)$, although differences were not statistically significant.

Figure 5 presents drug administration time for propofol, dexmedetomidine, and fentanyl in the ICU. Dexmedetomidine administration was prolonged in BiVP patients $(793 \pm$ 528 vs $478 \pm 295$ minutes; $P=.013)$. There was no significant difference in propofol $(497 \pm 328$ vs $394 \pm 331$ minutes; $P=.270)$ or fentanyl ( $802 \pm 545$ vs $547 \pm 352$ minutes; $P=.242$ ) duration. There was no significant difference in duration of ventilation between the groups $(1.37 \pm 1.05$ vs $1.34 \pm 1.72$ days; $P=.94)$.

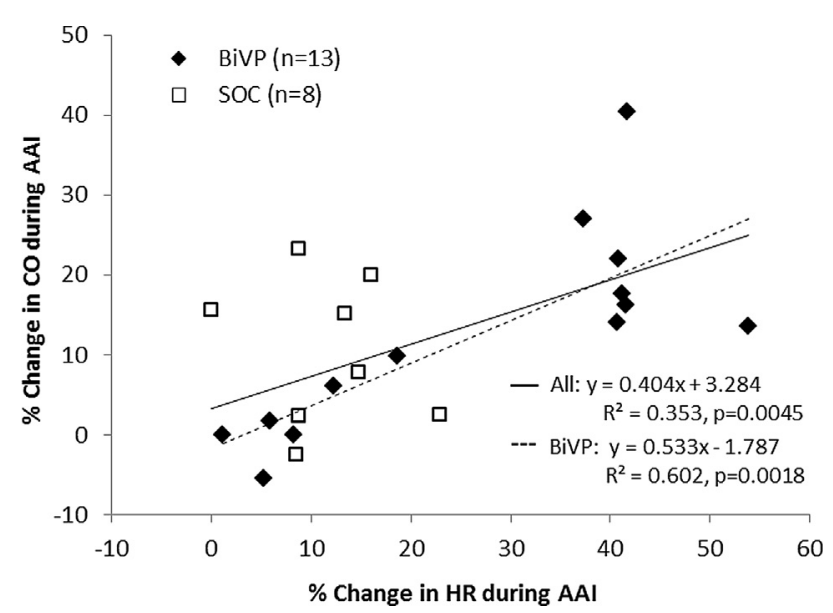

FIGURE 3. Percentage change in heart rate $(H R)$ and cardiac output $(C O)$ during atrial pacing $(A A I)$ at phase III. Regression lines are for all patients $(\mathrm{n}=21)$, and biventricular pacing $(B i V P)$ patients only $(\mathrm{n}=13)$ indicate the percentage change in HR is roughly twice the percentage change in CO. SOC, Standard of care. 


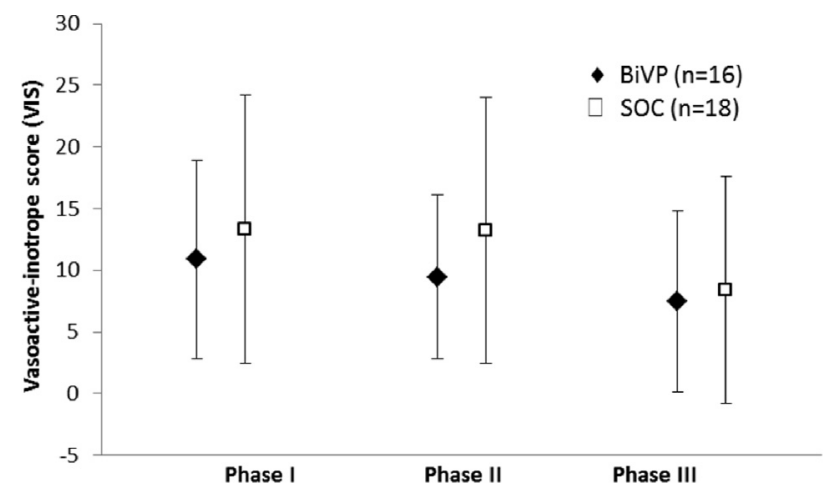

FIGURE 4. Mean vasoactive-inotrope score $(V I S)$ for biventricular pacing $(B i V P)$ and standard-of-care (SOC) groups. No difference in VIS was found in phases I, II, and III.

\section{DISCUSSION}

This substudy examines why AAI pacing becomes more effective over 12 to 24 hours after CPB relative to BiVP. ${ }^{17,18}$ One possibility is that decreasing intrinsic heart rates makes pacing-induced heart rate increases proportionately more important over time. Because the design of the BiPACS trial was based, in part, on the assumption that intrinsic heart rate would be similar in both study arms, this assumption requires confirmation.

In fact, we find a significantly lower intrinsic heart rate at phase III in the BiVP group, a difference not present in phases I and II. Furthermore, heart rate trended downward in BiVP patients from chest closure to 12 to 24 hours postoperatively.

Intrinsic heart rate in postoperative patients is affected by factors that are broadly divided into extrinsic stimuli, medication effects, and intrinsic autonomic effects. Extrinsic stimuli, such as pain, discomfort, and bedside activities, can increase arousal and intrinsic heart rate. ${ }^{20}$

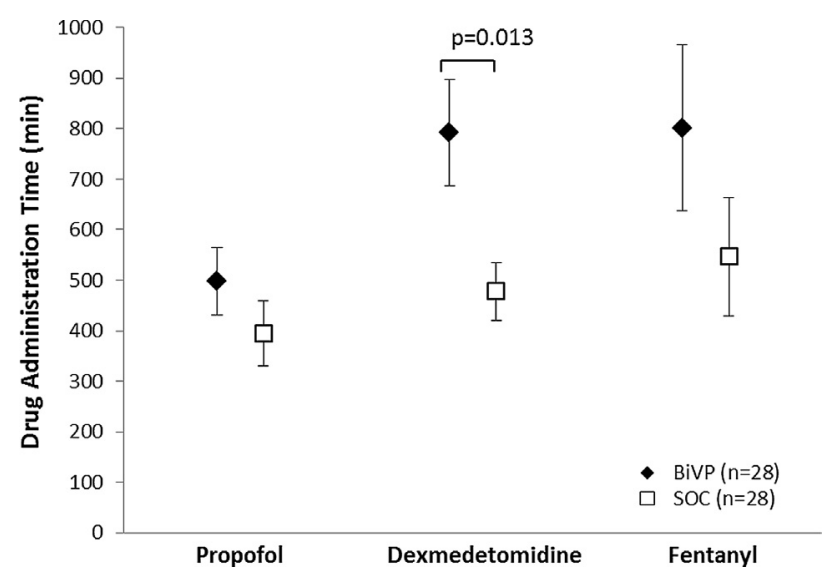

FIGURE 5. Mean administration time of propofol, dexmedetomidine, and fentanyl for biventricular pacing (BiVP) and standard-of-care (SOC) groups. BiVP patients were administered dexmedetomidine for significantly longer than SOC patients.
TABLE 3. Administration of vasoactive medications in phases I, II, and III

\begin{tabular}{llcl}
\hline & Phase I & Phase II & Phase III \\
\hline $\operatorname{BiVP}(\mathrm{n}=16)$ & $10.9 \pm 8.0$ & $9.7 \pm 6.7$ & $7.5 \pm 7.4$ \\
$\operatorname{SOC}(\mathrm{n}=18)$ & $13.3 \pm 10.9$ & $13.2 \pm 10.8$ & $8.4 \pm 9.2$ \\
\hline
\end{tabular}

$\mathrm{BiVP}$, Biventricular pacing; $S O C$, standard of care.

Medications include positive chronotropes, such as sympathomimetic amines and sedatives, which exert a negative chronotropic effect. ${ }^{21-23}$ Intrinsic autonomic effects are vagal or sympathetic, and can be affected by anxiety or feedback loops related to blood pressure, lactate levels, and atrial volume receptors. ${ }^{24}$

There are 3 potential explanations for the decreasing postoperative intrinsic heart rate observed in this study. First, improved hemodynamics during BiVP can reduce internal sympathetic drive. Trends in the BiVP group toward increasing $\mathrm{CO}$ and stroke volume from phase I to III are consistent with this concept and could reflect accelerated recovery from ischemia-reperfusion injury by BiVP. CO and stroke volume increase approximately $25 \%$ from phase I to phase III. Although BiVP can increase CO $15 \%$ to $20 \%$ compared with other forms of pacing, ${ }^{13}$ persistence of increased $\mathrm{CO}$ when pacing is turned off is more consistent with myocardial recovery than acute mechanical effects of pacing. Analysis of the primary end point of the BiPACS has addressed the overall effect of the pacing protocol. ${ }^{25}$

A second, related possibility is that reduced vasopressor and inotrope requirements decrease intrinsic heart rate in the BiVP group. A previous substudy from the BiPACS trial demonstrated reduced VIS in BiVP patients over 3 hours after CPB. ${ }^{19}$ How hemodynamics would influence the administration of vasoactive agents is undefined, however, and administration of these drugs is not regulated by protocol at Columbia. ${ }^{19}$ The present analysis demonstrates no significant difference in VIS between BiVP and SOC patients in phase III. Heart rate differences in phase III, therefore, probably do not reflect differences in vasoactive medication doses, although a small trend to decreased VIS in the BiVP group is present.

A third factor may be higher doses of sedatives in BiVP patients. $^{22,23}$ In fact, BiVP patients did receive dexmedetomidine for longer periods. Because there was little difference between groups in ventilation time, the reason for prolonged administration of dexmedetomidine in BiVP patients remains unexplained.

Stroke volume was significantly lower $(P=.020)$ in BiVP than SOC patients at phase I, indicating that the randomized groups were not precisely equivalent. In fact, mitral valve repair was more frequent in the BiVP group $(P=.04)$ and bypass time trended to be longer $(P=.08)$. Despite these differences, stroke volume increased significantly between phases I and III only in the BiVP group 
$(P=.051)$, suggesting clinical benefit. At phase III, there was no statistical difference between the BiVP and SOC groups in stroke volume $(P=.344)$.

Taken together, these factors are all likely to contribute to the lower heart rate observed in BiVP patients in the ICU. Lower intrinsic heart rate in BiVP patients may reflect reduced infusion of agents with chronotropic effects. This, in turn, could decrease frequency of atrial and ventricular arrhythmias and decrease requirements for antiarrhythmics. ${ }^{26}$

Our results support the view that lower heart rates augment the fractional increase in rate associated with pacing in the BiPACS trial. For BiVP patients, an average heart rate increase of $24 \%$ from 76.5 to $94.8 \mathrm{bpm}$ during AAI pacing is accompanied by an $11 \%$ increase in cardiac output from 4.4 to $4.9 \mathrm{~L} / \mathrm{min}$ (Table 2). This change matches the slope of the regressions in Figure 3. Therefore, the increased benefit of AAI pacing at phase III can be explained by decreased intrinsic heart rates. This observation applies only to the BiVP group. Similar effects are not seen in phase I, where increasing heart rate is associated with decreased stroke volume, and only BiVP pacing can augment cardiac output.

\section{Limitations}

The study is limited by missing data. For the 16 BiVP patients, heart rate data were available for 12 in phase I, 10 in phase II, and 13 in phase III. For the 18 SOC patients, heart rate data were available for 18 in phase I, 14 in phase II, and 9 in phase III. Thus, exclusion bias could confound the present results. Causes of missing data include heart block, which precluded measurement of intrinsic rate.

A clinical difference between randomization groups was a higher rate of mitral valve replacements and repairs in the $\mathrm{BiVP}$ group versus the SOC group. There were also trends toward longer bypass and crossclamp times for the BiVP group (Table 1). These trends were not associated with differences in intrinsic heart rate between groups in phases I and II (Figure 1). Stroke volume was significantly lower in BiVP than SOC patients at phase I. However, by phase III, there was no statistical difference in stroke volume between BiVP and SOC, suggesting a clinical benefit in the BiVP group leading to equalization between groups.

\section{CONCLUSIONS}

BiVP-randomized patients had lower intrinsic heart rates 12 to 24 hours after CPB compared with patients randomized to standard of care. This difference is further underscored by the observation that BiVP patients had a decreasing trend of intrinsic heart rates from immediately after chest closure to the ICU. These findings could be explained by a beneficial effect of optimized BiVP, as evidenced by increasing trends of cardiac output and stroke volume from after bypass to the ICU. However, the role of medications, such as vasopressors and inotropes, and heart rate-lowering medications, such as dexmedetomidine, needs to be further elucidated. Depression of intrinsic heart rate may contribute to increased benefit of heart rate acceleration by pacing in this clinical trial.

\section{References}

1. Cuculich PS, Joseph S. Cardiac resynchronization therapy: what? who? when? how? Am J Med. 2011;124:813-5.

2. Abraham WT, Fisher WG, Smith AL, Delurgio DB, Leon AR, Loh E, et al. Cardiac resynchronization in chronic heart failure. $N$ Engl J Med. 2002;346: 1845-53.

3. Cleland JG, Daubert JC, Erdmann E, Freemantle N, Gras D, Kappenberger L, et al. The effect of cardiac resynchronization on morbidity and mortality in heart failure. N Engl J Med. 2005;352:1539-49.

4. Tang AS, Wells GA, Talajic M, Arnold MO, Sheldon R, Connolly S, et al. Cardiac-resynchronization therapy for mild-to-moderate heart failure. $N \mathrm{Engl}$ J Med. 2010;363:2385-95.

5. St John Sutton M, Ghio S, Plappert T, Tavazzi L, Scelsi L, Daubert C, et al. Cardiac resynchronization induces major structural and functional reverse remodeling in patients with New York Heart Association Class I/II heart failure Circulation. 2009;120:1858-65.

6. Moss AJ, Hall WJ, Cannom DS, Klein H, Brown MW, Daubert JP, et al. Cardiacresynchronization therapy for the prevention of heart-failure events. $N$ Engl J Med. 2009;361:1329-38.

7. St John Sutton MG, Plappert T, Abraham WT, Smith AL, DeLurgio DB, Leon AR, et al. Effect of cardiac resynchronization therapy on left ventricular size and function in chronic heart failure. Circulation. 2003;107: 1985-90.

8. Nelson GS, Berger RD, Fetics BJ, Talbot M, Spinelli JC, Hare JM, et al. Left ventricular or biventricular pacing improves cardiac function at diminished energy cost in patients with dilated cardiomyopathy and left bundle-branch block. Circulation. 2000;102:3053-9.

9. Yau TM, Fedak PW, Weisel RD, Teng C, Ivanov J. Predictors of operative risk for coronary bypass operations in patients with left ventricular dysfunction. J Thorac Cardiovasc Surg. 1999;118:1006-13.

10. Antonio BL, Royster RL, Kon ND, Zvara DA. Acute biventricular pacing after cardiopulmonary bypass decreases myocardial dyssynchrony and increases cardiac index. J Cardiothorac Vasc Anesth. 2007;21:570-1.

11. Weisse $\mathrm{U}$, Isgro $\mathrm{F}$, Werling $\mathrm{CH}$, Lehmann $\mathrm{A}$, Saggau W. Impact of atrio-biventricular pacing to poor left-ventricular function after CABG. J Thorac Cardiovasc Surg. 2002;50:131-5.

12. Eberhardt F, Heringlake M, Massalme MS, Dyllus A, Misfeld M, Sievers HH et al. The effect of biventricular pacing after coronary artery bypass grafting: a prospective randomized trial of different pacing modes in patients with reduced left ventricular function. J Thorac Cardiovasc Surg. 2009;137:1461-7.

13. Straka F, Pirk J, Pindák M, Skalský I, Vančura V, Cihák R, et al. Biventricular pacing in the early postoperative period after cardiac surgery. Physiol Res. 2011;60:877-85.

14. Birnie DH, Tang AS. The problem of non-response to cardiac resynchronization therapy. Curr Opin Cardiol. 2006;21:20-6.

15. Stellbrink C, Breithardt OA, Franke A, Sack S, Bakker P, Auricchio A, et al. Impact of cardiac resynchronization therapy using hemodynamically optimized pacing on left ventricular remodeling in patients with congestive heart failure and ventricular conduction disturbances. J Am Coll Cardiol. 2001;38: 1957-65.

16. van Gelder BM, Bracke FA, Meijer A, Lakerveld LJ, Pijls NH. Effect of optimizing the VV interval on left ventricular contractility in cardiac resynchronization therapy. Am J Cardiol. 2004;93:1500-3.

17. Wang DY, Richmond ME, Quinn TA, Mirani AJ, Rusanov A, Yalamanchi V, et al Optimized temporary biventricular pacing acutely improves intraoperative cardiac output after weaning from cardiopulmonary bypass: a substudy of a randomized clinical trial. J Thorac Cardiovasc Surg. 2011;141:1002-8.

18. Rubinstein BJ, Wang DY, Cabreriza SE, Cheng B, Aponte-Patel L, Murata A et al. Response of mean arterial pressure to temporary biventricular pacing after chest closure during cardiac surgery. J Thorac Cardiovasc Surg. 2012;144: 1445-52.

19. Nguyen HV, Havalad V, Aponte-Patel L, Murata AY, Wang DY, Rusanov A, et al Temporary biventricular pacing decreases the vasoactive-inotrope score after 
cardiac surgery: a substudy of a randomized clinical trial. J Thorac Cardiovasc Surg. 2013;146:296-301.

20. Hu R, Stenson WG, Strichartz GR, Lilly LS. Mechanisms of cardiac arrhythmias. In: Lilly LS, ed. Pathophysiology of Heart Disease. 5th ed. Baltimore, MD: Lippincott Williams \& Wilkins; 2011:261-7.

21. Yamin CK, Miller CA, Antman EM, Strichartz GR, Lilly LS. Cardiovascular drugs. In: Lilly LS, ed. Pathophysiology of Heart Disease. 5th ed. Baltimore, MD: Lippincott Williams \& Wilkins; 2011:390-2, 406-8.

22. Heard C, Burrows F, Johnson K, Joshi P, Houck J, Lerman J. A comparison of dexmedetomidine-midazolam with propofol for maintenance of anesthesia in children undergoing magnetic resonance imaging. Pediatr Anesth. 2008;107: $1832-9$

23. Grounds RM, Venn RM. Comparison between dexmedetomidine and propofol for sedation in the intensive care unit: patient and clinician perceptions. Br J Anesth. 2001;87:684-90.

24. Costanzo LS. Physiology. 4th ed. Philadelphia, PA: Saunders Inc; 2010:134-5, 172-8.

25. Spotnitz HM, Cabreriza SE, Wang DY, Quinn TA, Cheng B, et al. Primary endpoints of the biventricular pacing after cardiac surgery trial. Ann Thorac Surg. 2013;96:808-15.

26. Mullany CJ. Coronary artery bypass surgery. Circulation. 2003;107:e21-2.

Access to The Journal of Thoracic and Cardiovascular Surgery Online is reserved for print subscribers!

Full-text access to The Journal of Thoracic and Cardiovascular Surgery Online is available for all print subscribers. To activate your individual online subscription, please visit The Journal of Thoracic and Cardiovascular Surgery Online, point your browser to http://www.mosby.com/itcvs, follow the prompts to activate your online access, and follow the instructions. To activate your account, you will need your subscriber account number, which you can find on your mailing label (note: the number of digits in your subscriber account number varies from 6 to 10). See the example below in which the subscriber account number has been circled:

\section{Sample mailing label}

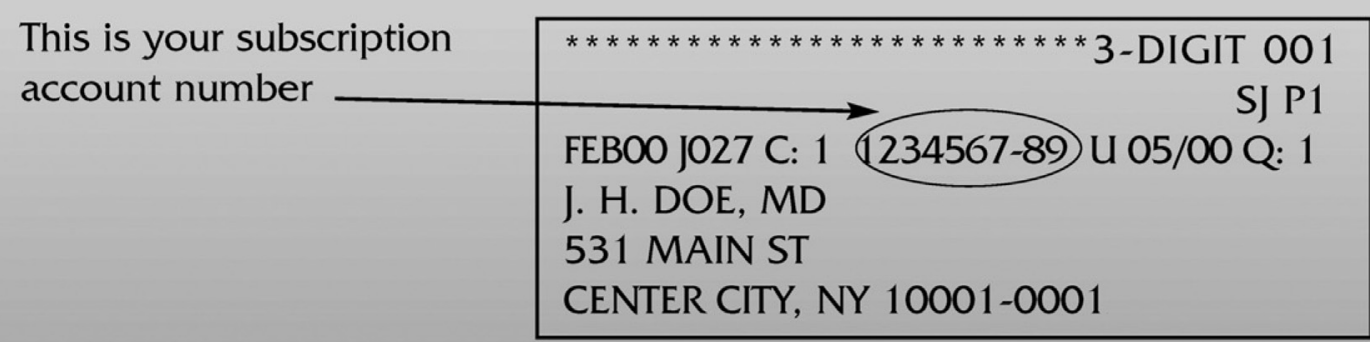

Personal subscriptions to The Journal of Thoracic and Cardiovascular Surgery Online are for individual use only and may not be transferred. Use of The Journal of Thoracic and Cardiovascular Surgery Online is subject to agreement to the terms and conditions as indicated online. 